\title{
Observation of Exclusive Electron-Positron Production in Hadron-Hadron Collisions
}

A. Abulencia, ${ }^{23}$ J. Adelman, ${ }^{13}$ T. Affolder, ${ }^{10}$ T. Akimoto,${ }^{55}$ M. G. Albrow, ${ }^{16}$ D. Ambrose, ${ }^{16}$ S. Amerio, ${ }^{43}$ D. Amidei, ${ }^{34}$ A. Anastassov, ${ }^{52}$ K. Anikeev,${ }^{16}$ A. Annovi, ${ }^{18} \mathrm{~J}$. Antos, ${ }^{1}$ M. Aoki,${ }^{55}$ G. Apollinari, ${ }^{16}$ J.-F. Arguin, ${ }^{33}$ T. Arisawa, ${ }^{57}$ A. Artikov, ${ }^{14}$ W. Ashmanskas, ${ }^{16}$ A. Attal, ${ }^{8}$ F. Azfar, ${ }^{42}$ P. Azzi-Bacchetta, ${ }^{43}$ P. Azzurri, ${ }^{46}$ N. Bacchetta, ${ }^{43}$ W. Badgett, ${ }^{16}$ A. Barbaro-Galtieri, ${ }^{28}$ V. E. Barnes, ${ }^{48}$ B. A. Barnett, ${ }^{24}$ S. Baroiant, ${ }^{7}$ V. Bartsch, ${ }^{30}$ G. Bauer,${ }^{32}$ F. Bedeschi, ${ }^{46}$ S. Behari, ${ }^{24}$ S. Belforte ${ }^{54}$ G. Bellettini, ${ }^{46}$ J. Bellinger, ${ }^{59}$ A. Belloni, ${ }^{32}$ D. Benjamin, ${ }^{15}$ A. Beretvas, ${ }^{16}$ J. Beringer, ${ }^{28}$ T. Berry, ${ }^{29}$ A. Bhatti, ${ }^{50}$ M. Binkley, ${ }^{16}$ D. Bisello, ${ }^{43}$ R. E. Blair, ${ }^{2}$ C. Blocker, ${ }^{6}$ B. Blumenfeld ${ }^{24}$ A. Bocci, ${ }^{15}$ A. Bodek, ${ }^{49}$ V. Boisvert ${ }^{49}$ G. Bolla ${ }^{48}$ A. Bolshov, ${ }^{32}$ D. Bortoletto, ${ }^{48}$ J. Boudreau, ${ }^{47}$ A. Boveia, ${ }^{10}$ B. Brau, ${ }^{10}$ L. Brigliadori, ${ }^{5}$ C. Bromberg,${ }^{35}$ E. Brubaker, ${ }^{13}$ J. Budagov, ${ }^{14}$ H. S. Budd, ${ }^{49}$ S. Budd, ${ }^{23}$ S. Budroni, ${ }^{46}$ K. Burkett, ${ }^{16}$ G. Busetto, ${ }^{43}$ P. Bussey, ${ }^{20}$ K. L. Byrum, S. Cabrera ${ }^{15}$ M. Campanelli, ${ }^{19}$ M. Campbell, ${ }^{34}$ F. Canelli, ${ }^{16}$ A. Canepa ${ }^{48}$ S. Carillo, ${ }^{17}$ D. Carlsmith,,${ }^{59}$ B. Caron, ${ }^{33}$ R. Carosi, ${ }^{46}$ M. Casarsa,${ }^{54}$ A. Castro, ${ }^{5}$ P. Catastini,${ }^{46}$ D. Cauz ${ }^{54}$ M. Cavalli-Sforza,${ }^{3}$ A. Cerri, ${ }^{28}$ L. Cerrito, ${ }^{42}$ S. H. Chang, ${ }^{27}$ Y. C. Chen, ${ }^{1}$ M. Chertok, ${ }^{7}$ G. Chiarelli, ${ }^{46}$ G. Chlachidze, ${ }^{14}$ F. Chlebana, ${ }^{16}$ I. Cho, ${ }^{27}$ K. Cho,${ }^{27}$ D. Chokheli, ${ }^{14}$ J. P. Chou, ${ }^{21}$ G. Choudalakis, ${ }^{32}$ S. H. Chuang, ${ }^{59}$ K. Chung, ${ }^{12}$ W. H. Chung, ${ }^{59}$ Y. S. Chung, ${ }^{49}$ M. Ciljak, ${ }^{46}$ C. I. Ciobanu, ${ }^{23}$ M. A. Ciocci, ${ }^{46}$ A. Clark,${ }^{19}$ D. Clark, ${ }^{6}$ M. Coca, ${ }^{15}$ G. Compostella, ${ }^{43}$ M. E. Convery, ${ }^{50}$ J. Conway, ${ }^{7}$ B. Cooper,${ }^{35}$ K. Copic,${ }^{34}$ M. Cordelli, ${ }^{18}$ G. Cortiana, ${ }^{43}$ F. Crescioli, ${ }^{46}$ C. Cuenca Almenar, ${ }^{7}$ J. Cuevas, ${ }^{11}$ R. Culbertson, ${ }^{16}$ J. C. Cully, ${ }^{34}$ D. Cyr, ${ }^{59}$ S. DaRonco, ${ }^{43}$ S. D’Auria, ${ }^{20}$ T. Davies,${ }^{20}$ M. D’Onofrio, ${ }^{3}$ D. Dagenhart, ${ }^{6}$ P. de Barbaro, ${ }^{49}$ S. De Cecco, ${ }^{51}$ A. Deisher,${ }^{28}$

G. De Lentdecker, ${ }^{49}$ M. Dell'Orso, ${ }^{46}$ F. Delli Paoli, ${ }^{43}$ L. Demortier, ${ }^{50}$ J. Deng, ${ }^{15}$ M. Deninno,,${ }^{5}$ D. De Pedis,${ }^{51}$ P. F. Derwent, ${ }^{16}$ G. P. Di Giovanni, ${ }^{44}$ C. Dionisi,${ }^{51}$ B. Di Ruzza, ${ }^{54}$ J. R. Dittmann, ${ }^{4}$ P. DiTuro, ${ }^{52}$ C. Dörr, ${ }^{25}$ S. Donati, ${ }^{46}$ M. Donega, ${ }^{19}$ P. Dong, ${ }^{8}$ J. Donini, ${ }^{43}$ T. Dorigo, ${ }^{43}$ S. Dube, ${ }^{52}$ J. Efron, ${ }^{39}$ R. Erbacher, ${ }^{7}$ D. Errede,${ }^{23}$ S. Errede, ${ }^{23}$ R. Eusebi, ${ }^{16}$ H. C. Fang, ${ }^{28}$ S. Farrington, ${ }^{29}$ I. Fedorko,${ }^{46}$ W. T. Fedorko, ${ }^{13}$ R. G. Feild, ${ }^{60}$ M. Feindt, ${ }^{25}$ J. P. Fernandez, ${ }^{31}$ R. Field, ${ }^{17}$ G. Flanagan, ${ }^{48}$ A. Foland, ${ }^{21}$ S. Forrester, ${ }^{7}$ G. W. Foster, ${ }^{16}$ M. Franklin, ${ }^{21}$ J. C. Freeman, ${ }^{28}$ I. Furic, ${ }^{13}$ M. Gallinaro, ${ }^{50}$ J. Galyardt, ${ }^{12}$ J. E. Garcia, ${ }^{46}$ F. Garberson, ${ }^{10}$ A. F. Garfinkel, ${ }^{48}$ C. Gay,${ }^{60}$ H. Gerberich,${ }^{23}$ D. Gerdes, ${ }^{34}$ S. Giagu ${ }^{51}$ P. Giannetti, ${ }^{46}$ A. Gibson, ${ }^{28}$ K. Gibson, ${ }^{47}$ J. L. Gimmell, ${ }^{49}$ C. Ginsburg, ${ }^{16}$ N. Giokaris, ${ }^{14}$ M. Giordani, ${ }^{54}$ P. Giromini, ${ }^{18}$ M. Giunta, ${ }^{46}$ G. Giurgiu, ${ }^{12}$ V. Glagolev ${ }^{14}$ D. Glenzinski, ${ }^{16}$ M. Gold,${ }^{37}$ N. Goldschmidt, ${ }^{17}$ J. Goldstein,${ }^{42}$ G. Gomez, ${ }^{11}$ G. Gomez-Ceballos, ${ }^{11}$ M. Goncharov, ${ }^{53}$ O. González, ${ }^{31}$ I. Gorelov, ${ }^{37}$ A. T. Goshaw, ${ }^{15}$ K. Goulianos, ${ }^{50}$ A. Gresele, ${ }^{43}$ M. Griffiths, ${ }^{29}$ S. Grinstein,${ }^{21}$ C. Grosso-Pilcher, ${ }^{13}$ R. C. Group, ${ }^{17}$ U. Grundler, ${ }^{23}$ J. Guimaraes da Costa, ${ }^{21}$

Z. Gunay-Unalan, ${ }^{35}$ C. Haber, ${ }^{28}$ K. Hahn, ${ }^{32}$ S. R. Hahn, ${ }^{16}$ E. Halkiadakis, ${ }^{52}$ A. Hamilton, ${ }^{33}$ B.-Y. Han, ${ }^{49}$ J. Y. Han, ${ }^{49}$ R. Handler ${ }^{59}$ F. Happacher,${ }^{18}$ K. Hara,${ }^{55}$ M. Hare,${ }^{56}$ S. Harper,${ }^{42}$ R. F. Harr,${ }^{58}$ R. M. Harris, ${ }^{16}$ M. Hartz, ${ }^{47}$

K. Hatakeyama ${ }^{50}$ J. Hauser, ${ }^{8}$ A. Heijboer, ${ }^{45}$ B. Heinemann, ${ }^{29}$ J. Heinrich,${ }^{45}$ C. Henderson, ${ }^{32}$ M. Herndon, ${ }^{59}$ J. Heuser ${ }^{25}$ D. Hidas, ${ }^{15}$ C. S. Hill, ${ }^{10}$ D. Hirschbuehl, ${ }^{25}$ A. Hocker,${ }^{16}$ A. Holloway, ${ }^{21}$ S. Hou, ${ }^{1}$ M. Houlden, ${ }^{29}$ S.-C. Hsu, ${ }^{9}$ B. T. Huffman, ${ }^{42}$ R. E. Hughes, ${ }^{39}$ U. Husemann,${ }^{60}$ J. Huston, ${ }^{35}$ J. Incandela,,${ }^{10}$ G. Introzzi,${ }^{46}$ M. Iori, ${ }^{51}$ Y. Ishizawa, ${ }^{55}$ A. Ivanov, ${ }^{7}$ B. Iyutin, ${ }^{32}$ E. James, ${ }^{16}$ D. Jang, ${ }^{52}$ B. Jayatilaka, ${ }^{34}$ D. Jeans, ${ }^{51}$ H. Jensen, ${ }^{16}$ E. J. Jeon, ${ }^{27}$ S. Jindariani, ${ }^{17}$ M. Jones, ${ }^{48}$ K. K. Joo, ${ }^{27}$ S. Y. Jun, ${ }^{12}$ J. E. Jung, ${ }^{27}$ T. R. Junk ${ }^{23}$ T. Kamon,${ }^{53}$ P. E. Karchin, ${ }^{58}$ Y. Kato, ${ }^{41}$ Y. Kemp, ${ }^{25}$ R. Kephart, ${ }^{16}$ U. Kerzel, ${ }^{25}$ V. Khotilovich, ${ }^{53}$ B. Kilminster, ${ }^{39}$ D. H. Kim, ${ }^{27}$ H. S. Kim, ${ }^{27}$ J. E. Kim, ${ }^{27}$ M. J. Kim, ${ }^{12}$ S. B. Kim, ${ }^{27}$ S. H. Kim, ${ }^{55}$ Y. K. Kim,${ }^{13}$ N. Kimura, ${ }^{55}$ L. Kirsch, ${ }^{6}$ S. Klimenko, ${ }^{17}$ M. Klute, ${ }^{32}$ B. Knuteson ${ }^{32}$ B. R. Ko, ${ }^{15}$ K. Kondo, ${ }^{57}$ D. J. Kong, ${ }^{27}$ J. Konigsberg, ${ }^{17}$ A. Korytov, ${ }^{17}$ A. V. Kotwal, ${ }^{15}$ A. Kovalev, ${ }^{45}$ A. C. Kraan, ${ }^{45}$ J. Kraus, ${ }^{23}$ I. Kravchenko, ${ }^{32}$ M. Kreps, ${ }^{25}$ J. Kroll, ${ }^{45}$ N. Krumnack, ${ }^{4}$ M. Kruse, ${ }^{15}$ V. Krutelyov, ${ }^{10}$ T. Kubo, ${ }^{55}$ S. E. Kuhlmann, ${ }^{2}$ T. Kuhr, ${ }^{25}$ Y. Kusakabe, ${ }^{57}$ S. Kwang, ${ }^{13}$ A. T. Laasanen, ${ }^{48}$ S. Lai, ${ }^{33}$ S. Lami, ${ }^{46}$ S. Lammel, ${ }^{16}$ M. Lancaster, ${ }^{30}$ R. L. Lander, ${ }^{7}$ K. Lannon, ${ }^{39}$ A. Lath, ${ }^{52}$ G. Latino, ${ }^{46}$ I. Lazzizzera, ${ }^{43}$ T. LeCompte,${ }^{2}$ J. Lee,${ }^{49}$ J. Lee,${ }^{27}$ Y. J. Lee,${ }^{27}$ S. W. Lee, ${ }^{53}$ R. Lefèvre, ${ }^{3}$

N. Leonardo, ${ }^{32}$ S. Leone, ${ }^{46}$ S. Levy, ${ }^{13}$ J. D. Lewis, ${ }^{16}$ C. Lin, ${ }^{60}$ C. S. Lin, ${ }^{16}$ M. Lindgren, ${ }^{16}$ E. Lipeles,,${ }^{9}$ A. Lister, ${ }^{7}$ D. O. Litvintsev, ${ }^{16}$ T. Liu, ${ }^{16}$ N. S. Lockyer ${ }^{45}$ A. Loginov, ${ }^{36}$ M. Loreti, ${ }^{43}$ P. Loverre, ${ }^{51}$ R.-S. Lu, ${ }^{1}$ D. Lucchesi, ${ }^{43}$ P. Lujan, ${ }^{28}$ P. Lukens, ${ }^{16}$ G. Lungu, ${ }^{17}$ L. Lyons,${ }^{42}$ J. Lys,${ }^{28}$ R. Lysak, ${ }^{1}$ E. Lytken, ${ }^{48}$ P. Mack, ${ }^{25}$ D. MacQueen, ${ }^{33}$ R. Madrak, ${ }^{16}$ K. Maeshima ${ }^{16}$ K. Makhoul, ${ }^{32}$ T. Maki,${ }^{22}$ P. Maksimovic,${ }^{24}$ S. Malde,${ }^{42}$ G. Manca ${ }^{29}$ F. Margaroli,${ }^{5}$ R. Marginean, ${ }^{16}$ C. Marino, ${ }^{25}$ C. P. Marino, ${ }^{23}$ A. Martin, ${ }^{60}$ M. Martin, ${ }^{20}$ V. Martin, ${ }^{20}$ M. Martínez, ${ }^{3}$ T. Maruyama, ${ }^{55}$ P. Mastrandrea, ${ }^{51}$ T. Masubuchi, ${ }^{55}$ H. Matsunaga, ${ }^{55}$ M.E. Mattson, ${ }^{58}$ R. Mazini, ${ }^{33}$ P. Mazzanti, ${ }^{5}$ K. S. McFarland, ${ }^{49}$ P. McIntyre, ${ }^{53}$ R. McNulty, ${ }^{29}$ A. Mehta, ${ }^{29}$ P. Mehtala ${ }^{22}$ S. Menzemer, ${ }^{11}$ A. Menzione, ${ }^{46}$ P. Merkel, ${ }^{48}$ C. Mesropian,${ }^{50}$ A. Messina, ${ }^{35}$ T. Miao, ${ }^{16}$ N. Miladinovic, ${ }^{6}$ J. Miles,${ }^{32}$ R. Miller,${ }^{35}$ C. Mills, ${ }^{10}$ M. Milnik, ${ }^{25}$ A. Mitra, ${ }^{1}$ G. Mitselmakher, ${ }^{17}$ A. Miyamoto, ${ }^{26}$ S. Moed,${ }^{19}$ N. Moggi,${ }^{5}$ B. Mohr,${ }^{8}$ R. Moore,${ }^{16}$ M. Morello, ${ }^{46}$ P. Movilla Fernandez, ${ }^{28}$ J. Mülmenstädt, ${ }^{28}$ A. Mukherjee, ${ }^{16}$

Th. Muller ${ }^{25}$ R. Mumford,${ }^{24}$ P. Murat,${ }^{16}$ J. Nachtman, ${ }^{16}$ A. Nagano, ${ }^{55}$ J. Naganoma,${ }^{57}$ I. Nakano, ${ }^{40}$ A. Napier,${ }^{56}$ 
V. Necula, ${ }^{17}$ C. Neu ${ }^{45}$ M. S. Neubauer, ${ }^{9}$ J. Nielsen ${ }^{28}$ T. Nigmanov, ${ }^{47}$ L. Nodulman, ${ }^{2}$ O. Norniella, ${ }^{3}$ E. Nurse,${ }^{30}$ S. H. Oh, ${ }^{15}$ Y.D. Oh,${ }^{27}$ I. Oksuzian, ${ }^{17}$ T. Okusawa,${ }^{41}$ R. Oldeman, ${ }^{29}$ R. Orava, ${ }^{22}$ K. Osterberg,${ }^{22}$ C. Pagliarone,${ }^{46}$ E. Palencia, ${ }^{11}$ V. Papadimitriou, ${ }^{16}$ A. A. Paramonov, ${ }^{13}$ B. Parks, ${ }^{39}$ S. Pashapour, ${ }^{33}$ J. Patrick, ${ }^{16}$ G. Pauletta, ${ }^{54}$ M. Paulini, ${ }^{12}$ C. Paus, ${ }^{32}$ D. E. Pellett, ${ }^{7}$ A. Penzo,${ }^{54}$ T. J. Phillips,${ }^{15}$ G. Piacentino, ${ }^{46}$ J. Piedra,${ }^{44}$ L. Pinera, ${ }^{17}$ J. Pinfold,${ }^{33}$ K. Pitts,${ }^{23}$ C. Plager, ${ }^{8}$ L. Pondrom, ${ }^{59}$ X. Portell, ${ }^{3}$ O. Poukhov, ${ }^{14}$ N. Pounder,${ }^{42}$ F. Prakoshyn,,${ }^{14}$ A. Pronko,,${ }^{16}$ J. Proudfoot,${ }^{2}$ F. Ptohos, ${ }^{18}$ G. Punzi, ${ }^{46}$ J. Pursley, ${ }^{24}$ J. Rademacker, ${ }^{42}$ A. Rahaman, ${ }^{47}$ N. Ranjan, ${ }^{48}$ S. Rappoccio, ${ }^{21}$ B. Reisert, ${ }^{16}$ V. Rekovic, ${ }^{37}$ P. Renton, ${ }^{42}$ M. Rescigno, ${ }^{51}$ S. Richter, ${ }^{25}$ F. Rimondi, ${ }^{5}$ L. Ristori, ${ }^{46}$ A. Robson, ${ }^{20}$ T. Rodrigo, ${ }^{11}$ E. Rogers,${ }^{23}$ S. Rolli, ${ }^{56}$ R. Roser, ${ }^{16}$ M. Rossi, ${ }^{54}$ R. Rossin, ${ }^{17}$ A. Ruiz, ${ }^{11}$ J. Russ, ${ }^{12}$ V. Rusu, ${ }^{13}$ H. Saarikko, ${ }^{22}$ S. Sabik, ${ }^{33}$ A. Safonov, ${ }^{53}$ W. K. Sakumoto, ${ }^{49}$ G. Salamanna, ${ }^{51}$ O. Saltó, ${ }^{3}$ D. Saltzberg, ${ }^{8}$ C. Sánchez, ${ }^{3}$ L. Santi, ${ }^{54}$ S. Sarkar,${ }^{51}$ L. Sartori,${ }^{46}$ K. Sato, ${ }^{16}$ P. Savard, ${ }^{33}$ A. Savoy-Navarro, ${ }^{44}$ T. Scheidle, ${ }^{25}$ P. Schlabach, ${ }^{16}$ E. E. Schmidt, ${ }^{16}$ M. P. Schmidt, ${ }^{60}$ M. Schmitt, ${ }^{38}$ T. Schwarz, ${ }^{7}$ L. Scodellaro, ${ }^{11}$ A. L. Scott, ${ }^{10}$ A. Scribano, ${ }^{46}$ F. Scuri,${ }^{46}$ A. Sedov, ${ }^{48}$ S. Seidel,${ }^{37}$ Y. Seiya,${ }^{41}$ A. Semenov,${ }^{14}$ L. Sexton-Kennedy, ${ }^{16}$ A. Sfyrla, ${ }^{19}$ M. D. Shapiro, ${ }^{28}$ T. Shears, ${ }^{29}$ P. F. Shepard, ${ }^{47}$ D. Sherman, ${ }^{21}$ M. Shimojima,${ }^{55}$ M. Shochet, ${ }^{13}$ Y. Shon, ${ }^{59}$ I. Shreyber, ${ }^{36}$ A. Sidoti, ${ }^{46}$ P. Sinervo, ${ }^{33}$ A. Sisakyan, ${ }^{14}$ J. Sjolin, ${ }^{42}$ A. J. Slaughter, ${ }^{16}$ J. Slaunwhite, ${ }^{39}$ K. Sliwa, ${ }^{56}$ J. R. Smith, ${ }^{7}$ F. D. Snider, ${ }^{16}$ R. Snihur, ${ }^{33}$ M. Soderberg, ${ }^{34}$ A. Soha, ${ }^{7}$ S. Somalwar, ${ }^{52}$ V. Sorin,${ }^{35}$ J. Spalding, ${ }^{16}$ F. Spinella, ${ }^{46}$ T. Spreitzer, ${ }^{33}$ P. Squillacioti, ${ }^{46}$ M. Stanitzki, ${ }^{60}$ A. Staveris-Polykalas, ${ }^{46}$ R. St. Denis, ${ }^{20}$ B. Stelzer, ${ }^{8}$ O. Stelzer-Chilton, ${ }^{42}$ D. Stentz, ${ }^{38}$ J. Strologas,${ }^{37}$ D. Stuart, ${ }^{10}$ J. S. Suh, ${ }^{27}$ A. Sukhanov, ${ }^{17}$ H. Sun, ${ }^{56}$ T. Suzuki, ${ }^{55}$ A. Taffard ${ }^{23}$ R. Takashima, ${ }^{40}$ Y. Takeuchi, ${ }^{55}$ K. Takikawa, ${ }^{55}$ M. Tanaka, ${ }^{2}$ R. Tanaka, ${ }^{40}$ M. Tecchio,${ }^{34}$ P. K. Teng, ${ }^{1}$ K. Terashi, ${ }^{50}$ J. Thom, ${ }^{16}$ A. S. Thompson, ${ }^{20}$ E. Thomson, ${ }^{45}$ P. Tipton, ${ }^{60}$ V. Tiwari, ${ }^{12}$ S. Tkaczyk, ${ }^{16}$ D. Toback, ${ }^{53}$ S. Tokar, ${ }^{14}$ K. Tollefson, ${ }^{35}$ T. Tomura, ${ }^{55} \mathrm{D}$. Tonelli, ${ }^{46} \mathrm{~S}$. Torre,${ }^{18} \mathrm{D}$. Torretta, ${ }^{16} \mathrm{~S}$. Tourneur,${ }^{44} \mathrm{~W}$. Trischuk,${ }^{33} \mathrm{R}$. Tsuchiya,${ }^{57}$ S. Tsuno, ${ }^{40}$ N. Turini, ${ }^{46}$ F. Ukegawa, ${ }^{55}$ T. Unverhau,${ }^{20}$ S. Uozumi,${ }^{55}$ D. Usynin, ${ }^{45}$ S. Vallecorsa, ${ }^{19}$ N. van Remortel, ${ }^{22}$ A. Varganov, ${ }^{34}$ E. Vataga,${ }^{37}$ F. Vázquez, ${ }^{17}$ G. Velev,${ }^{16}$ G. Veramendi, ${ }^{23}$ V. Veszpremi, ${ }^{48}$ R. Vidal, ${ }^{16}$ I. Vila, ${ }^{11}$ R. Vilar, ${ }^{11}$ T. Vine, ${ }^{30}$ I. Vollrath, ${ }^{33}$ I. Volobouev, ${ }^{28}$ G. Volpi, ${ }^{46}$ F. Würthwein, ${ }^{9}$ P. Wagner, ${ }^{53}$ R. G. Wagner, ${ }^{2}$ R. L. Wagner, ${ }^{16}$ J. Wagner, ${ }^{25}$ W. Wagner, ${ }^{25}$ R. Wallny, ${ }^{8}$ S. M. Wang, ${ }^{1}$ A. Warburton, ${ }^{33}$ S. Waschke, ${ }^{20}$ D. Waters, ${ }^{30}$ W. C. Wester III, ${ }^{16}$

B. Whitehouse, ${ }^{56}$ D. Whiteson, ${ }^{45}$ A. B. Wicklund, ${ }^{2}$ E. Wicklund, ${ }^{16}$ G. Williams, ${ }^{33}$ H. H. Williams, ${ }^{45}$ P. Wilson, ${ }^{16}$ B. L. Winer, ${ }^{39}$ P. Wittich, ${ }^{16}$ S. Wolbers,${ }^{16}$ C. Wolfe,${ }^{13}$ T. Wright,${ }^{34}$ X. Wu, ${ }^{19}$ S. M. Wynne, ${ }^{29}$ A. Yagil, ${ }^{16}$ K. Yamamoto, ${ }^{41}$ J. Yamaoka, ${ }^{52}$ T. Yamashita, ${ }^{40}$ C. Yang, ${ }^{60}$ U. K. Yang, ${ }^{13}$ Y. C. Yang, ${ }^{27}$ W. M. Yao, ${ }^{28}$ G. P. Yeh, ${ }^{16}$ J. Yoh, ${ }^{16}$ K. Yorita, ${ }^{13}$ T. Yoshida, ${ }^{41}$ G. B. Yu, ${ }^{49}$ I. Yu, ${ }^{27}$ S. S. Yu, ${ }^{16}$ J. C. Yun, ${ }^{16}$ L. Zanello, ${ }^{51}$ A. Zanetti, ${ }^{54}$ I. Zaw, ${ }^{21}$ X. Zhang, ${ }^{23}$ J. Zhou, ${ }^{52}$ and S. Zucchelli ${ }^{5}$

(CDF Collaboration)

${ }^{1}$ Institute of Physics, Academia Sinica, Taipei, Taiwan 11529, Republic of China

${ }^{2}$ Argonne National Laboratory, Argonne, Illinois 60439, USA

${ }^{3}$ Institut de Fisica d'Altes Energies, Universitat Autonoma de Barcelona, E-08193, Bellaterra (Barcelona), Spain ${ }^{4}$ Baylor University, Waco, Texas 76798, USA

${ }^{5}$ Istituto Nazionale di Fisica Nucleare, University of Bologna, I-40127 Bologna, Italy

${ }^{6}$ Brandeis University, Waltham, Massachusetts 02254, USA

${ }^{7}$ University of California, Davis, Davis, California 95616, USA

${ }^{8}$ University of California, Los Angeles, Los Angeles, California 90024, USA

${ }^{9}$ University of California, San Diego, La Jolla, California 92093, USA

${ }^{10}$ University of California, Santa Barbara, Santa Barbara, California 93106, USA

${ }^{11}$ Instituto de Fisica de Cantabria, CSIC-University of Cantabria, 39005 Santander, Spain

${ }^{12}$ Carnegie Mellon University, Pittsburgh, Pennsylvania 15213, USA

${ }^{13}$ Enrico Fermi Institute, University of Chicago, Chicago, Illinois 60637, USA

${ }^{14}$ Joint Institute for Nuclear Research, RU-141980 Dubna, Russia

${ }^{15}$ Duke University, Durham, North Carolina 27708

${ }^{16}$ Fermi National Accelerator Laboratory, Batavia, Illinois 60510, USA

${ }^{17}$ University of Florida, Gainesville, Florida 32611, USA

${ }^{18}$ Laboratori Nazionali di Frascati, Istituto Nazionale di Fisica Nucleare, I-00044 Frascati, Italy

${ }^{19}$ University of Geneva, CH-1211 Geneva 4, Switzerland

${ }^{20}$ Glasgow University, Glasgow G12 8QQ, United Kingdom

${ }^{21}$ Harvard University, Cambridge, Massachusetts 02138, USA

${ }^{22}$ Division of High Energy Physics, Department of Physics, University of Helsinki and Helsinki Institute of Physics, FIN-00014, Helsinki, Finland

${ }^{23}$ University of Illinois, Urbana, Illinois 61801, USA 


\author{
${ }^{24}$ The Johns Hopkins University, Baltimore, Maryland 21218, USA \\ ${ }^{25}$ Institut für Experimentelle Kernphysik, Universität Karlsruhe, 76128 Karlsruhe, Germany \\ ${ }^{26}$ High Energy Accelerator Research Organization (KEK), Tsukuba, Ibaraki 305, Japan \\ ${ }^{27}$ Center for High Energy Physics: Kyungpook National University, Taegu 702-701, Korea; \\ Seoul National University, Seoul 151-742, Korea; \\ and SungKyunKwan University, Suwon 440-746, Korea \\ ${ }^{28}$ Ernest Orlando Lawrence Berkeley National Laboratory, Berkeley, California 94720, USA \\ ${ }^{29}$ University of Liverpool, Liverpool L69 7ZE, United Kingdom \\ ${ }^{30}$ University College London, London WC1E 6BT, United Kingdom \\ ${ }^{31}$ Centro de Investigaciones Energeticas Medioambientales y Tecnologicas, E-28040 Madrid, Spain \\ ${ }^{32}$ Massachusetts Institute of Technology, Cambridge, Massachusetts 02139, USA \\ ${ }^{33}$ Institute of Particle Physics: University of Alberta, Edmonton, Canada T6G 2G7; \\ McGill University, Montréal, Canada H3A 2T8; \\ and University of Toronto, Toronto, Canada M5S 1A7 \\ ${ }^{34}$ University of Michigan, Ann Arbor, Michigan 48109, USA \\ ${ }^{35}$ Michigan State University, East Lansing, Michigan 48824, USA \\ ${ }^{36}$ Institution for Theoretical and Experimental Physics, ITEP, Moscow 117259, Russia \\ ${ }^{37}$ University of New Mexico, Albuquerque, New Mexico 87131, USA \\ ${ }^{38}$ Northwestern University, Evanston, Illinois 60208, USA \\ ${ }^{39}$ The Ohio State University, Columbus, Ohio 43210, USA \\ ${ }^{40}$ Okayama University, Okayama 700-8530, Japan \\ ${ }^{41}$ Osaka City University, Osaka 588, Japan \\ ${ }^{42}$ University of Oxford, Oxford OX1 3RH, United Kingdom \\ ${ }^{43}$ University of Padova, Istituto Nazionale di Fisica Nucleare, Sezione di Padova-Trento, I-35131 Padova, Italy \\ ${ }^{44}$ LPNHE, Universite Pierre et Marie Curie/IN2P3-CNRS, UMR7585, Paris, F-75252 France \\ ${ }^{45}$ University of Pennsylvania, Philadelphia, Pennsylvania 19104, USA \\ ${ }^{46}$ Istituto Nazionale di Fisica Nucleare Pisa, Universities of Pisa, Siena \\ and Scuola Normale Superiore, I-56127 Pisa, Italy \\ ${ }^{47}$ University of Pittsburgh, Pittsburgh, Pennsylvania 15260, USA \\ ${ }^{48}$ Purdue University, West Lafayette, Indiana 47907, USA \\ ${ }^{49}$ University of Rochester, Rochester, New York 14627, USA \\ ${ }^{50}$ The Rockefeller University, New York, New York 10021, USA \\ ${ }^{51}$ Istituto Nazionale di Fisica Nucleare, Sezione di Roma 1, University of Rome "La Sapienza," I-00185 Roma, Italy \\ ${ }^{52}$ Rutgers University, Piscataway, New Jersey 08855, USA \\ ${ }^{53}$ Texas A\&M University, College Station, Texas 77843, USA \\ ${ }^{54}$ Istituto Nazionale di Fisica Nucleare, University of Trieste/Udine, Italy \\ ${ }^{55}$ University of Tsukuba, Tsukuba, Ibaraki 305, Japan \\ ${ }^{56}$ Tufts University, Medford, Massachusetts 02155, USA \\ ${ }^{57}$ Waseda University, Tokyo 169, Japan \\ ${ }^{58}$ Wayne State University, Detroit, Michigan 48201, USA \\ ${ }^{59}$ University of Wisconsin, Madison, Wisconsin 53706, USA \\ ${ }^{60}$ Yale University, New Haven, Connecticut 06520, USA \\ (Received 27 November 2006; published 14 March 2007)
}

We present the first observation of exclusive $e^{+} e^{-}$production in hadron-hadron collisions, using $p \bar{p}$ collision data at $\sqrt{s}=1.96 \mathrm{TeV}$ taken by the run II Collider Detector at Fermilab, and corresponding to an integrated luminosity of $532 \mathrm{pb}^{-1}$. We require the absence of any particle signatures in the detector except for an electron and a positron candidate, each with transverse energy $E_{T}>5 \mathrm{GeV}$ and pseudorapidity $|\eta|<2$. With these criteria, 16 events are observed compared to a background expectation of $1.9 \pm 0.3$ events. These events are consistent in cross section and properties with the QED process $p \bar{p} \rightarrow$ $p+e^{+} e^{-}+\bar{p}$ through two-photon exchange. The measured cross section is $1.6_{-0.3}^{+0.5}$ (stat) \pm 0.3 (syst) $\mathrm{pb}$. This agrees with the theoretical prediction of $1.71 \pm 0.01 \mathrm{pb}$.

In this Letter we report an observation of exclusive dilepton production in hadron-hadron collisions. In an exclusive dilepton process there are no particles produced other than the lepton pair, and the incident hadrons do not dissociate. The process $\gamma \gamma \rightarrow \ell^{+} \ell^{-}$in hadron collisions is discussed in Ref. [1]. In this Letter we consider $p \bar{p} \rightarrow p+$ $e^{+} e^{-}+\bar{p}$, where the electron pair is produced via $\gamma \gamma \rightarrow$ $e^{+} e^{-}$. The events are simulated using the LPAIR Monte Carlo generator [2].

Exclusive events have been proposed as a search channel for new physics at the Large Hadron Collider (LHC) [3] because, if the momentum of the outgoing protons is 
measured, exclusive processes provide an additional method to calculate the invariant mass of the central system [4]. Also, since the interaction depends only on quantum electrodynamics and the proton electromagnetic form factor at small momentum transfer, the cross section is known with an accuracy better than $1 \%$. This makes exclusive dilepton processes potentially interesting candidates for improving the typical 5\% uncertainty on the luminosity measurement at hadron colliders [5]. Exclusive dilepton events can provide an excellent control sample for other exclusive processes whose theoretical predictions are less certain, such as exclusive diphoton producion [6] and exclusive Higgs production [3]. At the LHC, exclusive dilepton events may also be used to calibrate forward proton spectrometers [4]. Two-photon production of lepton pairs has been experimentally observed in $e p$ [7], and $A A$ [8] collisions, but the exclusive process has never been observed in hadron-hadron collisions. There were indications of exclusive muon pair production at the intersecting storage rings [9].

The present analysis uses a data set corresponding to an integrated luminosity $\mathcal{L}=532 \pm 32 \mathrm{pb}^{-1}$ collected by the run II Collider Detector at Fermilab (CDF II) [10]. CDF II is a general purpose particle detector at the Tevatron, a $p \bar{p}$ collider with center of mass energy $\sqrt{s}=1.96 \mathrm{TeV}$. The experimental signature of exclusive $e^{+} e^{-}$production is an $e^{+} e^{-}$pair with no other particles detected. The proton and antiproton lose a small fraction of their momentum in these collisions and escape along the beam direction without being detected.

We briefly describe the components of CDF II that are used in this analysis [11]. Both the central calorimeter [12], covering $|\eta|<1.1$, and the plug calorimeter [13], covering $1.1<|\eta|<3.6$, have separate electromagnetic (leadscintillator) and hadronic (steel-scintillator) compartments. The energy resolution for the central electromagnetic calorimeter is $\sigma(E) / \sqrt{E}=13.5 \% \oplus 1.5 \%$, and for the plug electromagnetic calorimeter it is $\sigma(E) / \sqrt{E}=$ $16 \% \oplus 1 \%$. The central electromagnetic calorimeters contain strip chambers that measure the transverse profile of the electromagnetic shower at a depth of six radiation lengths. The electromagnetic calorimeters are also capable of determining the arrival time of a shower in the calorimeter with a resolution of $0.6 \mathrm{~ns}$ [14]. The miniplug calorimeter [15] is a lead-liquid scintillator sampling calorimeter and covers the region $3.6<|\eta|<5.2$. Covering $5.4<|\eta|<7.4$ is a series of three scintillator paddles on each side of the detector, collectively called the beam shower counters (BSC), labeled BSC1, BSC2, and BSC3, in order of increasing $|\eta|$. BSC1 is preceded by 2 radiation lengths of lead.

Inside the calorimeter is a tracking system composed of a solenoid producing a $1.4 \mathrm{~T}$ magnetic field, the silicon tracking detector, and the central outer tracker (COT) [16]. The tracking system measures charged particle tracks in $|\eta|<1.0$ with an efficiency near unity, and charged particle tracks in $1.0<|\eta|<2.8$ with reduced efficiency.

The data were collected using a trigger requiring two clusters [17] of energy in the central or plug electromagnetic calorimeters and no activity in the BSC1 counters. Events containing two electron candidates with $E_{T}>$ $5 \mathrm{GeV}$ and $|\eta|<2.0$ are selected. Here, and in the remainder of this Letter, we use the term "electron" to mean either electron or positron candidate. Electron candidates are defined as clusters in the electromagnetic calorimeter with a hadronic-to-electromagnetic energy ratio and lateral shower shape consistent with an electron, and a track with $p_{T}>1 \mathrm{GeV} / c$ pointing to the calorimeter cluster. The efficiency for triggering, reconstructing, and identifying both electrons in a signal event is $\varepsilon_{\mathrm{ee}}=(26 \pm 3) \%$. A large contribution to $\varepsilon_{\mathrm{ee}}$ comes from the electron trigger efficiency, $(77 \pm 5) \%$ per electron, due to the trigger turnon at the threshold.

Events caused by cosmic ray interactions occur at random times, so we require both clusters to have a measurement in the electromagnetic calorimeter timing system with times that are consistent with the collision. The overall efficiency for the cosmic rejection requirement is $\varepsilon_{\text {cosmic }}=(93 \pm 3) \%$.

To select exclusive events, we require that no additional particle signatures be detected in the calorimeters or BSC. A particle signature in the calorimeter is defined as a cluster of adjacent towers above the noise threshold in the miniplug, or a single tower above the noise threshold in the plug and central calorimeters. An additional cluster is defined as a cluster that is not part of either electron in the event. A particle signature in the BSC is defined as any hit above the noise threshold of the BSC.

When an inelastic interaction occurs in the same bunch crossing as an exclusive event, it will deposit energy in the calorimeters and $\mathrm{BSC}$, preventing the identification of the exclusive event. This efficiency $\varepsilon_{\text {exc }}$ is the fraction of zerobias events (triggered solely on the bunch crossing time) that pass the exclusivity selection divided by the total number of zero-bias events. This is directly related to the instantaneous bunch luminosity [18]. Using the same run range for the zero-bias data sample as the exclusive $e^{+} e^{-}$ triggered events, the overall exclusive efficiency is $\varepsilon_{\text {exc }}=$ $8.6 \%$, with negligible statistical uncertainty. The systematic uncertainty on this fraction is negligible compared to the uncertainty of the luminosity.

As a consequence of the exclusivity selection requirements, electrons that emit sufficient bremsstrahlung (final state radiation, FSR) to deposit energy outside the electron's cluster will be excluded from the candidate sample. The corresponding efficiency is calculated by generating events with the LPAIR simulation plus the GEANT-based [19] detector simulation, then requiring the events to pass the exclusivity selection requirements. This efficiency is $\varepsilon_{\mathrm{FSR}}=(79 \pm 5) \%$. The dominant source of uncertainty in $\varepsilon_{\mathrm{FSR}}$ is the material count in the detector. 
The overall efficiency to identify exclusive $e^{+} e^{-}$events with the luminosity distribution of this data sample is $(1.6 \pm 0.2) \%$. This is dominated by $\varepsilon_{\text {exc }}$, which is low because of the large fraction of bunch crossings with more than one inelastic $p \bar{p}$ interaction.

A total of 16 candidate events pass electron identification, cosmic ray rejection, and exclusivity requirements. All of these events contain oppositely charged electron pairs. The invariant mass and $\Delta \phi$ (the opening angle between the $e^{+}$and $e^{-}$in $r-\phi$ plane) distributions of the candidate sample are compared to events generated with LPAIR plus detector simulation in Fig. 1. Other variables, such as the $p_{T}$ of the $e^{+} e^{-}$pair and $\eta$ of the electrons are also in agreement with LPAIR.

There are four backgrounds to consider: jets that pass electron requirements (jet fakes), cosmic rays that interact in the detector, nonexclusive events, and $\gamma \gamma \rightarrow e^{+} e^{-}$ events with proton dissociation.

The jet fake background consists of exclusive events with a hadronic final state, such as $\pi^{+} \pi^{-}$, that fake a signal event when both hadrons are reconstructed in the detector as electrons. In a jet-triggered sample we observe no events that pass the exclusivity cuts but fail the electron requirements. This results in an upper limit on the rate of this process. Multiplying this upper limit with the measured probability of a generic calorimeter cluster to pass the electron cuts, an upper limit on the jet fake background is determined to be 0.1 events. Thus the jet fake background is taken to be $0.0_{-0.0}^{+0.1}$ events.

By examining the rate of out-of-time cosmic ray events, the probability that a candidate event originates from a cosmic ray is found to be negligible.

The third background is due to nonexclusive events in which one or more particles passed undetected through cracks in the calorimeter, or were below the noise thresholds, causing the event to appear exclusive. The multiplicity of additional clusters is shown in Fig. 2(a). A clear peak is observed in the zero additional clusters signal region. To estimate the amount of background in the signal region, the events with 5 to 50 additional clusters were fit to the function $y=e^{(a x+b)}$, where the result of the fit yields $a=0.05 \pm 0.01$ and $b=-1.3 \pm 0.4$. The fit result predicts a nonexclusive background of $0.3 \pm 0.1$ events with zero additional clusters.
To verify that a peak in the signal region is not expected with inclusive events, we plot the number of associated clusters for a sample of $Z \rightarrow e^{+} e^{-}$events selected from the same run range as the exclusive $e^{+} e^{-}$data set (the $Z$ boson cannot be produced exclusively at a hadron collider due to Yang's theorem [20]) as well as a sample of inclusive Drell-Yan events, simulated with the PYTHIA [21] Monte Carlo generator, in Fig. 2(b). The $Z \rightarrow e^{+} e^{-}$data sample contains events with two electrons that have an invariant mass between $81 \mathrm{GeV} / c^{2}$ and $101 \mathrm{GeV} / c^{2}$ and a single vertex reconstructed in the event. There is no peak at zero additional clusters in either the $Z \rightarrow e^{+} e^{-}$sample nor the Drell-Yan simulated sample. We also verified that this distribution is nearly independent of the dielectron invariant mass value using the simulated Drell-Yan sample.

The dissociation background arises from events that are mediated by two-photon exchange, but instead of being truly exclusive, one or both protons are excited into a lowmass state that dissociates. There is a small probability that these dissociations will escape detection in the BSC, and hence would not be distinguished from exclusive events. We use two simulation programs, GRAPE-DILEPTON [22] and LPAIR, to estimate this background.

The LPAIR (GRAPE-DILEPTON) simulation predicts that $7 \%(5 \%)$ of proton dissociations will have all dissociation products too far forward to be detectable. The average of the two estimates, $(6 \pm 1) \%$, is taken as the probability that a dissociated proton will escape detection. To estimate the number of dissociation background events in the candidate sample, the cross sections for events in which one or both protons dissociate are extracted from LPAIR [23] and then multiplied by the probability that the dissociation will escape detection. The number of dissociation events in the 16 event signal sample is estimated to be $1.6 \pm 0.3$. Therefore, the sum of all background sources is $N_{\mathrm{bkgd}}=$ $1.9 \pm 0.3$ events.

The cross section $\sigma$ for exclusive $e^{+} e^{-}$production with $E_{T}>5 \mathrm{GeV}$ and $|\eta|<2$ is calculated using

$$
\sigma=\frac{N_{\mathrm{data}}-N_{\mathrm{bkgd}}}{\varepsilon_{\mathrm{cosmic}} \varepsilon_{\mathrm{FSR}} \varepsilon_{\mathrm{ee}} \varepsilon_{\mathrm{exc}} \mathcal{L}} .
$$

Considering that the exclusive process is derived from the experimental observation (the sum of the exclusive and the
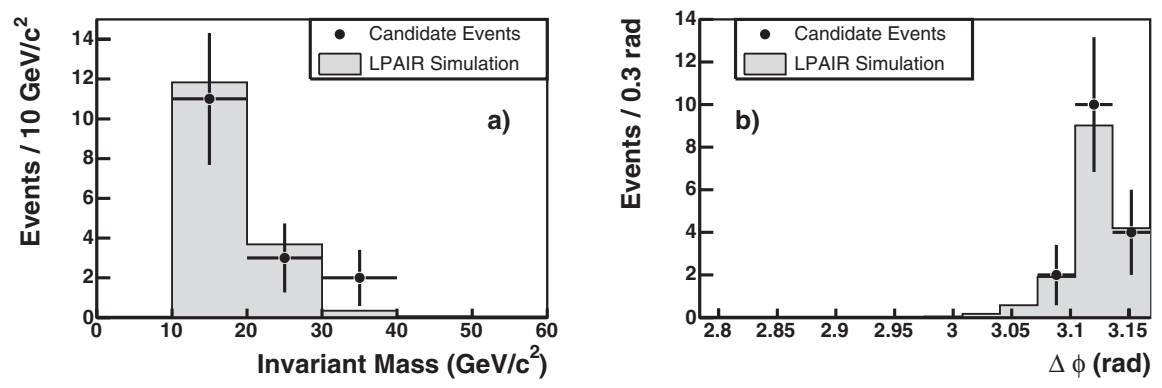

FIG. 1. The 16 candidate events (points) compared to the LPAIR simulation (solid). (a) Invariant mass of $e^{+} e^{-}$ pair (resolution $\sim 8 \%$ ), (b) $\Delta \phi$ between $e^{+} e^{-}$pair. The simulation is normalized to the number of data events. 

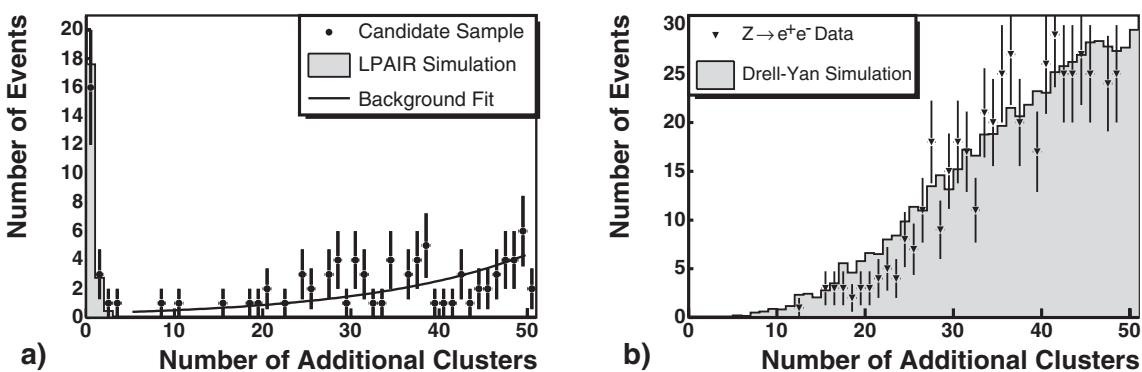

FIG. 2. (a) The number of additional clusters in LPAIR simulation and signal sample events with all selection criteria applied except the exclusivity requirement. LPAIR simulation events are normalized to the number of data events with less than 5 clusters. The line shows the fit used for the background estimate. (b) The number of additional clusters for $Z \rightarrow e^{+} e^{-}$data and Drell-Yan simulation, the simulation is normalized to the number of data events.

disassociation processes) we report both the total and the exclusive cross sections.

The cross section for exclusive $p \bar{p} \rightarrow p+e^{+} e^{-}+\bar{p}$ is measured to be $1.6_{-0.3}^{+0.5}$ (stat) \pm 0.3 (syst) pb using $N_{\text {bkgd }}=$ $1.9 \pm 0.3$ events. This agrees with the theoretical cross section $1.71 \pm 0.01 \mathrm{pb}$ given by LPAIR. The probability of observing 16 events when $1.9 \pm 0.3$ events are expected is $1.3 \times 10^{-9}$, equivalent to a $5.5 \sigma$ effect [24].

The measured cross section for $\gamma \gamma \rightarrow e^{+} e^{-}$, where all the proton and antiproton dissociation products are contained within $|\eta|>7.4$, is measured to be $1.8_{-0.3}^{+0.5}$ (stat) \pm 0.2 (syst) pb using $N_{\text {bkgd }}=0.3 \pm 0.1$ events (the sum of all backgrounds except the dissociation). This agrees with the theoretical prediction of $1.9 \pm 0.4$ (syst) pb for this cross section, determined using LPAIR. The uncertainty on this prediction is larger than for the purely exclusive LPAIR prediction because assumptions about the hadronization of the dissociating proton are made in this estimate.

In conclusion, we have observed 16 exclusive electron pair events in CDF II, with $|\eta|<2.0$ and $E_{T}>5 \mathrm{GeV}$, with a background estimate of $1.9 \pm 0.3$ events. These events are consistent in both their cross section and kinematic distributions with $p \bar{p} \rightarrow p+e^{+} e^{-}+\bar{p}$ through two-photon exchange $\left(\gamma \gamma \rightarrow e^{+} e^{-}\right)$. This is the first time that exclusive two-photon processes have been observed in hadron-hadron collisions, and implies that the LHC experiments can rely on this process to calculate expectations for new physics and luminosity measurements.

We thank the Fermilab staff and the technical staffs of the participating institutions for their vital contributions. This work was supported by the U.S. Department of Energy and National Science Foundation; the Italian Istituto Nazionale di Fisica Nucleare; the Ministry of Education, Culture, Sports, Science and Technology of Japan; the Natural Sciences and Engineering Research Council of Canada; the National Science Council of the Republic of China; the Swiss National Science Foundation; the A.P. Sloan Foundation; the Bundesministerium für Bildung und Forschung, Germany; the Korean Science and Engineering Foundation and the Korean Research
Foundation; the Particle Physics and Astronomy Research Council and the Royal Society, UK; the Russian Foundation for Basic Research; the Comisión Interministerial de Ciencia y Tecnología, Spain; in part by the European Community's Human Potential Programme under Contract No. HPRN-CT-2002-00292; and the Academy of Finland.

[1] H. Bhabha, Proc. R. Soc. A 152, 559 (1935); V. Budnev et al., Phys. Lett. B 39, 526 (1972).

[2] J. Vermaseren, Nucl. Phys. B229, 347 (1983).

[3] M.G. Albrow and A. Rostovtsev, hep-ph/0009336; K. Piotrzkowski, Phys. Rev. D 63, 071502 (2001); V. Khoze et al., Eur. Phys. J. C 23, 311 (2002); A. Kaidalov et al., Eur. Phys. J. C 31, 387 (2003); A. Kaidalov et al., Eur. Phys. J. C 33, 261 (2004); V. Khoze et al., Eur. Phys. J. C 34, 327 (2004).

[4] CERN Report No. CERN-LHCC-2005-025; LHC Report No. LHCC-I-015.

[5] B. Caron and J. Pinfold, Helsinki 2000, Workshop on Forward Physics and Luminosity at the LHC; V. Khoze et al., Eur. Phys. J. C 19, 313 (2001); A. Shamov and V. Telnov, Nucl. Instrum. Methods Phys. Res., Sect. A 494, 51 (2002); D. Bocian and K. Piotrzkowski, Acta Phys. Pol. B 35, 2417 (2004).

[6] A. Abulencia et al., Phys. Rev. Lett. (to be published).

[7] A. Aktas et al., Phys. Lett. B 583, 28 (2004); A. Aktas et al., Eur. Phys. J. C 31, 17 (2003).

[8] A. Belkacem et al., Phys. Rev. A 56, 2806 (1997); C. Vane et al., Phys. Rev. A 50, 2313 (1994); R. Baur et al., Phys. Lett. B 332, 471 (1994); J. Adams et al., Phys. Rev. C 70, 031902 (2004).

[9] D. Antreasyan et al., CERN, Report No. CERN-EP/80-82, 1980 (unpublished).

[10] D. Acosta et al., Phys. Rev. D 71, 032001 (2005).

[11] CDF II uses a cylindrical coordinate system with the $z$ coordinate along the proton beam direction, in which $\theta$ is the polar angle, $\phi$ is the azimuthal angle, and pseudorapidity $\eta=-\ln \tan (\theta / 2) . E_{T}=E \sin \theta$ and $p_{T}=p \sin \theta$, where $E$ is the energy measured by the calorimeter and $p$ the momentum measured in the tracking system. 
[12] L. Balka et al., Nucl. Instrum. Methods Phys. Res., Sect. A 267, 272 (1988); S. Bertolucci et al., Nucl. Instrum. Methods Phys. Res., Sect. A 267, 301 (1988).

[13] M. Albrow et al., Nucl. Instrum. Methods Phys. Res., Sect. A 480, 524 (2002).

[14] M. Goncharov et al., FERMILAB, Report No. FERMILAB-PUB-05-543-E, 2005; Nucl. Instrum. Methods Phys. Res., Sect. A 565, 543 (2006).

[15] M. Gallinaro et al., IEEE Trans. Nucl. Sci. 52, 879 (2005).

[16] T. Affolder et al., Nucl. Instrum. Methods Phys. Res., Sect. A 526, 249 (2004).

[17] The calorimeter is constructed of many separate segments, called towers. A cluster is defined as a set of adjacent towers with an appreciable amount of energy deposited in them.

[18] Bunch luminosity is the instantaneous luminosity of each of the 36 individual bunch crossings at the Tevatron.
[19] GEANT, detector description and simulation tool, CERN Program Library Long Writeup W5013 (1993).

[20] C. N. Yang, Phys. Rev. 77, 242 (1950).

[21] T. Sjöstrand et al., Comput. Phys. Commun. 135, 238 (2001). We use version 2.616.

[22] T. Abe, Comput. Phys. Commun. 136, 126 (2001).

[23] The LPAIR simulation only describes the kinematics of the dissociating proton, so the Minimum Bias Rockefeller (MBR) simulation [25] is used to simulate the products of the proton dissociation.

[24] This probability is calculated from the sum of Poisson probabilities for seeing the observed number of events or more, convoluted with a Gaussian for the expected number of background events.

[25] F. Abe et al., Phys. Rev. D 50, 5535 (1994). 